\title{
LA REFORMA DEL PROCESO DE SELECCIÓN Y NOMBRAMIENTO DE LOS MAGISTRADOS DEL TRIBUNAL SUPREMO DE CANADÁ: EL CASO PARTICULAR DE QUEBEC
}

\author{
FRANCISCO JAVIER ROMERO CARO \\ Investigador postdoctoral \\ Universidad del País Vasco
}

TRC, núm. 46, 2020, pp. 541-560

ISSN $1139-5583$

\begin{abstract}
SUMARIO
I. Introducción. II. La regulación jurídico-constitucional del Tribunal Supremo. Breves apuntes. III. Composición, elegibilidad y nombramiento: el rol del Parlamento federal. IV. La reforma del proceso de selección y nombramiento de los magistrados del Tribunal Supremo. V. La participación provincial en el proceso de selección: el caso particular de Quebec. VI. Reflexiones finales.
\end{abstract}

\section{INTRODUCCIÓN}

Los procesos de selección de los magistrados del intérprete supremo de la Constitución - ya sea en forma de Tribunal Supremo o Tribunal Constitucional- suelen entrañar un elevado grado de complejidad. Esta aumenta especialmente en contextos de elevada polarización política hasta el extremo de que los mismos pueden resultar en una fuerza lesiva para la legitimidad de dicha institución que menoscabe el sistema democrático ${ }^{1}$.

1 La crítica política a la labor del Tribunal Supremo no es un fenómeno ajeno al modelo canadiense, siendo esta de especial intensidad por parte del movimiento soberanista quebequés tras los dictámenes acerca de la patriación constitucional (Reference re Resolution to Amend the Constitution, [1981] 1 S.C.R. 753) y el derecho de veto por parte de Quebec (Re: Objection by Quebec to a Resolution to amend the Constitution, [1982] 2 S.C.R. 793), calificando al mismo como la Cour des autres (el Tribunal de los otros) en referencia a la mayoría anglófona de Canadá. 
Una vez resueltas por el Tribunal Supremo una serie de cuestiones relativas a la naturaleza constitucional de la institución y los requisitos de elegibilidad de sus miembros, este confirmó la necesidad de llevar a cabo una reforma constitucional en caso de que se pretenda modificar alguno de sus elementos esenciales. En consecuencia, dada la rigidez de la Constitución de Canadá y la imposibilidad práctica para concluir una reforma con éxito, quedó patente que cualquier modificación del régimen jurídico de la institución debía versar sobre aspectos que pudieran ser reformados por vías infra-constitucionales. Siguiendo dicha línea, el gobierno de Justin Trudeau ha puesto en marcha una serie de reformas del proceso de selección y nombramiento de los magistrados del Tribunal Supremo de Canadá con el objetivo de aumentar la transparencia y la rendición de cuentas de un proceso, hasta hora, dominado totalmente por el ejecutivo.

La reforma del proceso de selección y nombramiento también ha servido para articular la participación de Quebec en el mismo, dando traslado a una de las demandas tradicionales de la provincia, que no pudo ser materializada debido al fracaso de las rondas constitucionales del Lago Meech y Charlottetown. Aunque esta no satisfaga completamente las demandas de la provincia francófona, el nuevo sistema supone un importante avance en pos del acomodo de Quebec en el seno de la federación canadiense con potencial para extenderse a otras instituciones.

\section{LA REGULACIÓN JURÍDICO-CONSTITUCIONAL DEL TRIBUNAL SUPREMO. BREVES APUNTES}

La Constitución de Canadá, ya sea el texto de 1867 o el de 1982, no contiene una regulación expresa del Tribunal Supremo². La única mención a una institución de dicha naturaleza es la contenida en el artículo 101 de la Constitución de 1867, que autoriza al Parlamento federal a crear una Corte de Apelación General (General Court of Appeal) o cualquier otro tribunal que considere oportuno para una mejor administración judicial en Canadá3.

No será hasta 1875 cuando se cree el Tribunal Supremo ${ }^{4}$, una institución que, paradójicamente, carecía de supremacía en el ordenamiento jurídico canadiense, siendo la última instancia judicial el Comité Judicial del Consejo Privado de la Reina (Judicial Committee of the Privy Council), sito en Londres. Esta falta de supremacía se veía acrecentada por la posibilidad de, previo acuerdo de las partes, renunciar a su jurisdicción y apelar los fallos de los tribunales provinciales

2 La creación de la Confederación supuso el mantenimiento del régimen de tribunales que existía en las colonias tal como se recoge en el art. 129 de la Constitución de 1867.

3 HogG; Constitutional Law of Canada, Carswell, Toronto, 2007: 4-2.

4 Supreme and Exchequer Courts Act, S.C. 1875, c. 11. 
directamente al Privy Council's. En 1949, se aprueba una reforma de la Supreme Court Act $(\mathrm{SCA})^{6}$ mediante la que se abolió definitivamente la competencia del Privy Council en materia civil, proceso que se completará diez años más tarde con la resolución del último litigio pendiente ${ }^{7}$. Dicha reforma situó al Tribunal Supremo en la cúspide del sistema judicial canadiense, convirtiéndolo en la última instancia judicial de Canadá, siendo, por tanto, sus decisiones inapelables ante cualquier otro tribunal.

El Tribunal Supremo se encuentra regulado en una ley ordinaria, la Supreme Court Act ${ }^{8}$. Dado que dicha norma no se encuentra entre los textos a los que el artículo 52 de la Constitución de 1982 dota de rango constitucional, nos encontramos, en principio, ante una institución de naturaleza infraconstitucional ${ }^{9}$. Sin embargo, a pesar de que la Constitución de Canadá no contenga una regulación expresa del Tribunal Supremo, sí que recoge —en los artículos 41 y 42- la necesidad de seguir unos determinados procedimientos de reforma constitucional para variar algunos aspectos del mismo. En particular, debe seguirse el procedimiento general de reforma del artículo 38 - que exige la aprobación del Parlamento federal y de al menos 7 provincias, siempre que estas agrupen, como mínimo, al 50\% de la población de la federación- para modificar cualquier aspecto que afecte al Tribunal Supremo, a excepción de la composición del mismo, materia que se encuentra especialmente protegida requiriendo de unanimidad provincial para su reforma ${ }^{10}$. Estos artículos dotan al Tribunal Supremo de una naturaleza particular, pues estamos ante la única materia no contemplada en la Constitución de Canadá que requiere seguir los procedimientos constitucionales de reforma para ser modificada ${ }^{11}$.

La inclusión del Tribunal Supremo entre las materias contempladas en los artículos 41 y 42 conlleva la paradoja de que sea necesario reformar la Constitución, incluso por el procedimiento agravado que exige la unanimidad provincial, para modificar aspectos de una institución que no se encuentra regulada en la misma. En consecuencia, la modificación de determinados aspectos de una ley

5 MCCORMicK; Supreme at Last: The Evolution of the Supreme Court of Canada, James Lorimer \& Company Ltd, Toronto, 2000, p. 6.

6 Act to amend the Supreme Court Act, S.C. 1949 (2nd Sess.), c. 37, ss. 1, 3.

7 La abolición de la competencia penal del Privy Council se produjo en 1888, aunque fue declarada inválida en 1926 pues el Parlamento de Canadá no podía modificar una norma imperial. La misma se materializará definitivamente en 1933 tras la aprobación del Estatuto de Westminster, siendo el último caso resuelto por el Privy Council Ponoka-Calmar Oils Ltd. v Earl F. Wakefield Co. (Canada) [1959] UKPC 20.

8 Actualmente se encuentra vigente una norma reformada tras la patriación denominada Supreme Court Act (R.S.C., 1985, c. S-26).

9 Newman; «The Constitutional Status of the Supreme Court of Canada», Supreme Court Law Review, n. ${ }^{\circ} 47$ (2d), 2009, pp. 429-443.

10 El art. 42.1.d establece el procedimiento general como mecanismo para la reforma de la institución, con la excepción de lo establecido en el art. 41.d, el cual hace referencia a la composición del tribunal.

11 Pelletier; «Les modalités de la modification de la Constitution du Canada», Revue Juridique Thémis, n. ${ }^{\circ} 33,1999$, pp. 5-58. 
ordinaria, la Supreme Court Act, no puede ser llevada a cabo por el Parlamento federal de manera autónoma, sino que requiere de la aprobación provincial que exija el procedimiento aplicable de reforma constitucional. Esta paradoja tiene su fundamento en la dificultad para reconciliar un sistema parlamentario de base británica —asentado sobre un concepto político de Constitución- con una Constitución — como concepto jurídico- concebida como norma suprema, tensionando las costuras del sistema constitucional canadiense. A pesar de lo anterior, existe cierta controversia en la doctrina canadiense acerca de la aplicabilidad de los artículos 41 y 42 al Tribunal Supremo. Mientras que autores como Hogg sostienen que cualquier aspecto de la Supreme Court Act puede ser modificado por el Parlamento federal, incluso llegando a la abolición de la institución, derogando la norma, dada su condición de ley ordinaria ${ }^{12}$, otros como Monahan y Shaw estiman que dicho razonamiento llevaría a considerar dichos preceptos constitucionales como meramente virtuales e inaplicables, dado que se refieren a materias no contempladas hasta la fecha en el texto constitucional ${ }^{13}$.

La cuestión referente a la naturaleza constitucional de la Supreme Court Act fue zanjada por el Tribunal Supremo en 2014, a raíz del contencioso sobre la elegibilidad del juez Marc Nadon para formar parte del Tribunal Supremo ${ }^{14}$. En su dictamen, los magistrados reconocieron el «estatus constitucional» del Tribunal Supremo, una vez constituido como cúspide del sistema tras la abolición de las apelaciones al Privy Council ${ }^{15}$. Esta decisión supuso una expansión del ámbito material de la Constitución de Canadá, la cual va más allá del límite formal recogido en el artículo 52 de la Constitución de $1982^{16}$. De igual modo, confirmaron que cualquier variación de los elementos esenciales del Tribunal requiere de una reforma constitucional siguiendo el procedimiento general de reforma del artículo $38^{17}$, rechazando, igualmente, que el Parlamento federal tenga capacidad

12 Hogg; 2007, op. cit: 4-14. Este autor considera que los arts. 41 y 42 de la Constitución de 1982 únicamente serán de aplicación en caso de una futura inclusión expresa de la composición y funciones del Tribunal Supremo en la Constitución.

13 Monahan y Shaw; Constitutional Law, Fourth Edition, Irwin Law, Toronto, 2013, p. 205.

14 Reference re Supreme Court Act, ss. 5 and 6, 2014 SCC 21, [2014] 1 S.C.R. 433, en adelante, Supreme Court Reference. Vid. nota 26.

15 Ibíd., par. 95. Sobre este «estatus constitucional» vid. KARAZIVAN; «De la structure constitutionnelle dans le Renvoi relatif au Sénat: vers une gestalt constitutionnelle?», McGill Law Journal, Vol. 60, No.4, 2015, pp. 793-838.

16 Peach; «Reference re Supreme Court Act, ss 5 and 6 - Expanding the Constitution of Canada», Constitutional Forum, Vol. 23, n. ${ }^{\circ}$ 1, 2014, pp. 1-6. No es descartable que la superación de la rigidez formal del artículo 52 dé lugar a nuevas ampliaciones de los límites constitucionales para incluir otras normas de especial trascendencia constitucional como la relativa a las lenguas oficiales (Official Languages Act (R.S.C., 1985 , c. 31 (4th Supp.))).

17 Aunque el Tribunal no especificó cuáles son los dichos «elementos esenciales» sí que estableció que entre ellos se encuentran la posición del Tribunal Supremo como cúspide del sistema judicial canadiense o las funciones de intérprete constitucional (Supreme Court Reference, par. 94). Monahan y Shaw (2013): 205 estiman que los mecanismos de nombramiento y remoción de jueces también deberían ser considerados como elementos esenciales de la institución. 
para alterar la composición del Tribunal, pues dicha circunstancia requiere reformar la Constitución por el procedimiento de unanimidad tal como dicta el artículo $41^{18}$. Por lo tanto, la referencia que se hace en los artículos 41 y 42 al Tribunal Supremo no es meramente virtual (empty vessels) a la espera de la inclusión de una regulación de la institución en la Constitución ${ }^{19}$, sino que es sustantiva, siendo de plena aplicación, pues responde a la voluntad del constituyente de 1982 de otorgar una protección reforzada al Tribunal Supremo con el fin de evitar su modificación unilateral por parte del Parlamento federal ${ }^{20}$. Esta consideración supone reducir la competencia del Parlamento federal a aquellos elementos no esenciales de la Supreme Court Act, meramente destinados a asegurar el mantenimiento de la institución, que pueden ser modificados a través del procedimiento legislativo ordinario ${ }^{21}$.

\section{COMPOSICIÓN, ELEGIBILIDAD Y NOMBRAMIENTO: EL ROL DEL PARLAMENTO FEDERAL}

El Tribunal Supremo de Canadá está integrado por nueve magistrados, de los que uno debe ejercer como presidente ${ }^{22}$. Al menos tres de ellos deben provenir de la provincia de Quebec — artículo 6 SCA — dado el sistema de derecho civil propio de dicha provincia — producto de su pasado colonial—, aspecto que ha convertido a Canadá en un estado con dualidad jurídica en el que conviven el common law de origen británico y el code civil de ascendencia francesa ${ }^{23}$. Aunque la Supreme Court Act no contiene ningún ulterior requisito en relación con la composición territorial del Tribunal Supremo, la práctica política ha derivado en la convención de nombrar tres jueces de Ontario, dos de las provincias del oeste y uno de las atlánticas. La edad de retiro obligatorio está fijada a los 75 años, aunque es posible $-\mathrm{y}$ frecuente- la jubilación anticipada.

La elegibilidad de los miembros del Tribunal se encuentra regulada en los artículos 5 y 6 de la Supreme Court Act, siendo estos criterios diferentes para los

18 Supreme Court Reference, par. 74.

19 Este aspecto ha sido reiterado por el Tribunal Supremo en oposición al argumento del Fiscal General, quien consideraba vacíos de contenido los preceptos del título V de la Constitución de 1982 relativos al Tribunal Supremo. Supreme Court Reference, párrafos 97-103.

20 Supreme Court Reference, par. 92.

21 Ibíd., par. 101. Esta interpretación está estrechamente conectada con la realizada en el Re: Authority of Parliament in relation to the Upper House, (1980) 1 S.C.R. 54, ahondando en la restricción judicial de los poderes unilaterales del Parlamento federal a la hora de modificar el sistema institucional.

22 Art. 4.1 Supreme Court Act. En sus inicios el Tribunal estaba compuesto por seis miembros, de los que dos debían provenir de Quebec. En 1927 se amplió a siete, creciendo la cuota de Quebec a tres (An Act to amend the Supreme Court Act, S.C. 1927, c. 38, s. 1). La composición actual data de 1949, manteniéndose en tres la representación de la provincia francófona (An Act to amend the Supreme Court Act, S.C. 1949, c. 37, s.1).

23 Brown-JoHn y PAwLEY; "When legal systems meet: bijuralism in the Canadian federal system», BCN Political Science Debates, 2005, n. ${ }^{\circ}$, pp. 147-170. 
magistrados nombrados por la cuota de Quebec. Con carácter general, y a excepción del caso de Quebec, cualquier persona que sea o haya sido juez de un Tribunal Superior provincial es elegible como miembro del Tribunal Supremo. En el caso de los abogados, se requiere que el candidato sea o haya sido miembro del Colegio de la provincia durante al menos diez años. En el caso de la cuota perteneciente a Quebec la regulación legal es diferente, siendo elegibles los miembros de la Corte Superior (Cour supérieure) y de la Corte de Apelación de Quebec (Cour d'appel), así como a aquellos abogados que estén colegiados en la provincia, sin que sea de aplicación ningún límite temporal ${ }^{24}$.

En lo referente al nombramiento, formalmente este se produce por el Gobernador General a propuesta del Consejo de Ministros (artículo 4.2 SCA), sin que sea necesaria, a diferencia de lo que ocurre en EE.UU. ${ }^{25}$, su confirmación por parte del Parlamento. Tampoco hay previsión legal alguna respecto a la necesidad de consultar a los gobiernos de las provincias, de manera que el poder de nombramiento reside exclusivamente en el poder ejecutivo ${ }^{26}$. Por otro lado, es habitual que el Ministro de Justicia realice consultas informales - las cuales generalmente tienen carácter confidencial - con juristas de reconocido prestigio o, incluso, con el Presidente del Tribunal Supremo acerca de la idoneidad de los potenciales candidatos que baraje el ejecutivo. Otro factor presente en el proceso de nombramiento es la representatividad de las dos tradiciones jurídicas de Canadá, la del common law y la civilista. Esta cuestión es de especial importancia a la hora de elegir al Presidente del Tribunal — quien también es designado por el Primer Ministro- primando la misma sobre el criterio de antigüedad ${ }^{27}$, existiendo la costumbre de alternar la presidencia del Tribunal Supremo entre jueces provenientes de las dos tradiciones jurídicas ${ }^{28}$.

Aunque no haya obligación legal alguna de recabar el consentimiento del Parlamento federal para proceder con el nombramiento, la participación de dicha institución en el proceso de selección y nombramiento de los integrantes del Tribunal Supremo ha sido una cuestión controvertida en los últimos años.

24 Esta diferente regulación fue objeto de controversia tras el nombramiento de Marc Nadon —un juez supranumerario de la Corte Federal de Apelaciones que formó parte en el pasado al Colegio de Abogados de Quebec- como miembro del TS por el cupo de Quebec. Finalmente, el TS consideró inválido dicho nombramiento al entender que únicamente son elegibles por la cuota de Quebec aquellos abogados colegiados en la provincia actualmente en ejercicio, El Tribunal justificó su decisión en la inmutabilidad del contenido del artículo 6 desde 1886, así como en la finalidad del mismo, que no es otra que asegurar la supervivencia de la tradición civilista de Quebec, así como los valores que la misma entraña y representa (Supreme Court Reference, par 17).

25 El inciso dos de la sección segunda del artículo 2 de la Constitución de los EE.UU. supedita el nombramiento realizado por el Presidente al consentimiento del Senado.

26 Morton; «Judicial Appointments in the Post-Charter Era: A system in Transition» en MALLESON y Russell, (eds.); Appointing Judges in an Age of Judicial Power. Critical Perspectives from around the World, University of Toronto Press, Toronto, 2006, pp. 56-79.

27 Esta costumbre se ha mantenido de manera ininterrumpida desde 1933 a excepción de la designación de Dickson en 1984 por parte de Pierre E. Trudeau.

28 Tras la jubilación de la Presidenta McLachlin en 2017, perteneciente al sistema de common law, la presidencia recayó en el juez Wagner, proveniente de la tradición civilista de Quebec, en un nuevo ejemplo de la fuerza vinculante que ejerce esta convención entre la clase política. 
A pesar de que tradicionalmente el nombramiento era decidido de forma unilateral por el Primer Ministro — previo paso a ser aprobado por el gobiernosin intervención parlamentaria alguna, esta práctica comenzó a variar en 2004 cuando, por primera vez, se pusieron en marcha mecanismos de participación parlamentaria con el objetivo de reducir el sesgo mayoritario derivado de la nominación por parte del ejecutivo ${ }^{29}$. Dicho mecanismo consistió en la comparecencia del Ministro de Justicia ante una comisión de la Cámara de los Comunes con el fin de informar sobre las razones que habían llevado al gobierno a seleccionar a los jueces Abella y Charron como nuevos integrantes del Tribunal Supremo ${ }^{30}$. Esta comparecencia, en la que no participaron los candidatos propuestos, no fue bien recibida por la oposición conservadora al estimar que la misma solo servía para maquillar la falta de transparencia del proceso de nombramiento, rechazando apoyar ninguno de los nombramientos ${ }^{31}$. Pese al relativo fracaso de esta comparecencia parlamentaria, la misma supuso un hito en la historia de Canadá pues por primera vez el Parlamento participaba, si bien a título meramente informativo, en el proceso de designación. En 2005, tras la jubilación del juez Major, el gobierno liberal de Paul Martin creó un panel de expertos, entre los que se encontraban un parlamentario por cada partido ${ }^{32}$, que debía examinar una lista de ocho candidatos con el objetivo de seleccionar al más idóneo para el puesto, que el gobierno se comprometía a designar. Este proceso no llegó a cristalizar debido a que el ejecutivo fue derrotado en una moción de censura poco después de que le fuera propuesta una terna final, celebrándose nuevas elecciones.

Harper decidió continuar con el proceso iniciado por su antecesor, eligiendo a uno de los integrantes de la terna propuesta, el juez Rothstein. El Primer Ministro condicionó dicho nombramiento a que Rothstein compareciera ante una comisión parlamentaria creada ad hoc y respondiera a las cuestiones que los diputados le formularan ${ }^{33}$, siguiendo el modelo de confirmación imperante en el Senado de los EE.UU. Esta audiencia no conllevó ningún tipo de voto, más allá de que los integrantes del mismo compartieran sus impresiones con el Ministro de Justicia. Tras la celebración de la misma, el juez Rothstein fue formalmente nominado. Esta audiencia parlamentaria supuso una novedad en el parlamentarismo canadiense, teniendo una gran aceptación. Aunque el

29 Whyte; «Political Accountability in Appointments to the Supreme Court of Canada», Constitutional Forum, Vol. 25, n. ${ }^{\circ}$ 3, 2016, pp. 109-118.

30 Hogg, «Appointment of Justice Marshall Rothstein to the Supreme Court of Canada», Osgoode Hall Law Journal, Volume 44, n. ${ }^{\circ}$ 3, 2006, pp. 527-538.

31 Songer; The Transformation of the Supreme Court of Canada. An Empirical Examination, University of Toronto Press, Toronto, 2008.

32 Junto a ellos también había un representante de las sociedades jurídicas provinciales y dos personas de distinguida trayectoria profesional que fueran ajenas a la abogacía y la judicatura.

33 La comisión fue denominada Ad Hoc Committee to Review a Nominee for the Supreme Court of Canada, estando compuesta por diputados de todos los partidos según su representación proporcional en la Cámara de los Comunes. 
resultado de la comisión no era jurídicamente vinculante, políticamente sí que podía entenderse como tal, pues una mala acogida por parte de los integrantes de esta hubiera puesto en cuestión la idoneidad el nombramiento.

La nominación de Rothstein parecía abrir un camino hacia un mayor control parlamentario de los procesos de nombramiento de los magistrados del Tribunal Supremo, aumentando la transparencia y legitimidad de los mismos. Cuando en 2008 se produjo una nueva vacante, todo hacía indicar que se seguiría el mismo proceso. Sin embargo, Harper decidió prescindir del panel de expertos dada las críticas de la oposición a la composición del mismo. Además, una convocatoria de elecciones anticipadas a finales de 2008 suspendió indefinidamente la comparecencia del juez Cromwell ante el Parlamento, regresándose al sistema de nombramientos tradicio$\mathrm{nal}^{34}$. Una vez transcurrido el periodo electoral y aliviada la tensión política, el reelegido gobierno de Harper decidió reabrir el proceso de nombramiento a la participación del Parlamento. Las audiencias ante una comisión parlamentaria fueron reinstauradas en los nombramientos de Moldaver y Karakatsanis (2011), Wagner (2012), así como en el frustrado nombramiento de Nadon (2013). El rechazo al nombramiento de este último provocó que el gobierno federal regresara de nuevo al sistema de designación unilateral sin intervención del Parlamento como muestra de disconformidad por la decisión del Tribunal Supremo. De esta manera, no se celebraron comparecencias parlamentarias durante los procesos de nombramiento de Gascon y Côté (2014) y Brown (2015) como integrantes del Tribunal Supremo.

Por su parte, el gobierno liberal de Justin Trudeau retomó la práctica previa como parte de sus compromisos de transparencia y mejora de la calidad democrática. El nombramiento de Malcom Rowe (2016) como integrante del Tribunal fue precedido de una comparecencia pública ante una comisión parlamentaria, integrada tanto por diputados como senadores, en la que fue cuestionado sobre su trayectoria como juez, así como por su visión en relación con los desafíos a los que se enfrenta el Tribunal; idéntico proceso al que se sometieron Sheilah Martin (2017) y Nicholas Kasirer (2019). En todo caso, aunque la participación del Parlamento en el proceso de nombramiento ha ido tímidamente abriéndose paso, esta no ha llegado a consolidarse dada la ausencia de un procedimiento reglado, aspecto que ha resultado en que la misma quede al arbitrio del gobierno de turno en función de la coyuntura política.

\section{LA REFORMA DEL PROCESO DE SELECCIÓN Y NOMBRAMIENTO DE LOS MAGISTRADOS DEL TRIBUNAL SUPREMO}

Dentro del paquete de medidas destinadas a mejorar la calidad democrática, el gobierno de Trudeau decidió, durante el verano de 2016, iniciar un proceso de

34 Hogg; (2013): Appointment of Thomas A. Cromwell to the Supreme Court of Canada en en Verrelli (ed.); The Democratic Dilemma: Reforming Canada's Supreme Court, Institute of Intergovernmental Relations, Montreal \& Kingston, 2016, pp. 13-25. 
reforma de algunos aspectos del proceso de selección y nombramiento de los magistrados del Tribunal Supremo. Siguiendo la misma filosofía aplicada en lo concerniente al Senado ${ }^{35}$, el ejecutivo liberal procedió a crear un panel de expertos —denominado Independent Advisory Board for Supreme Court of Canada Judicial Appointments - encargado de identificar a aquellas personas cualificadas para el puesto que, además, sean funcionalmente bilingües y representen la diversidad que caracteriza a la sociedad canadiense.

El panel de expertos, inspirado en el modelo existente en el Reino Unido ${ }^{36}$, está compuesto por siete miembros con un mandato máximo, renovable, de hasta cinco años tal como queda establecido en su norma reguladora ${ }^{37}$. De los siete integrantes del panel, tres son nombrados por el gobierno federal, a propuesta del Ministro de Justicia, de los cuales, al menos dos, no pueden ser abogados o procuradores. Los cuatro miembros restantes son nombrados por organizaciones ajenas al ejecutivo. Tanto a la Canadian Bar Association, el Colegio de Abogados federal, como a la Federation of Law Societies of Canada, agrupación que aúna a todas las asociaciones de juristas provinciales y territoriales, les corresponde nombrar a un abogado en ejercicio de reconocido prestigio. Por su parte, el Canadian Judicial Council, el Consejo Judicial Canadiense, debe nombrar a un juez superior ya jubilado, mientras que al Consejo de Decanos de Derecho corresponde nombrar a un jurista de reconocido prestigio. La presidencia del Independent Advisory Board será ejercida por uno de sus siete integrantes, nombrado a tal efecto por el gobierno federal, condición que actualmente recae en la antigua Primera Ministra Kim Campbell.

Con la clara finalidad de potenciar la independencia del panel en consonancia con los objetivos pretendidos por el nuevo sistema - aumentar la transparencia y la rendición de cuentas-, los miembros nombrados por el ejecutivo federal no constituyen mayoría en este ${ }^{38}$. La preponderancia de los representantes procedentes de las principales ramas del mundo jurídico —abogados, jueces y académicos-, quienes deben gozar de una acreditada trayectoria, ha resultado en una

35 Apenas unos meses antes, el gobierno federal puso en marcha un nuevo método de selección de los senadores con el objetivo de incrementar su independencia y legitimidad a ojos de la ciudadanía. Para un análisis de la misma, vid. Romero CARo; "Canadá. La reforma del Senado como vía de mejora de la calidad democrática y la representación territorial» en Tudela, Kölling y Reviriego (coords.); Calidad Democrática y Organización Territorial, Marcial Pons, Madrid, 2018, pp. 243-260.

36 Malleson, «The New Judicial Appointments Commission in England and Wales: New Wine in Old Bottles?» en Malleson y Russell, (eds.); Appointing Judges in an Age of Judicial Power. Critical Perspectives from around the World, University of Toronto Press, Toronto, 2006, pp. 39-55.

37 Order in Council PC 2016-0693. Hasta la fecha, el ejecutivo federal ha decidido establecer la duración del mandato en seis meses, con el objetivo de ir renovando a la mayoría de los integrantes del panel con excepción de la presidenta.

38 Dentro de las medidas orientadas a aumentar la transparencia, el Tribunal Supremo celebró a finales de septiembre de 2019 dos vistas de apelaciones en Winnipeg (Manitoba) con la finalidad de acercar el funcionamiento de la justicia a la sociedad. Ello constituye un hito pues es la primera vez en la historia que esta institución se reúne fuera de su sede en Ottawa desde su creación en 1875. 
primacía de los criterios profesionales sobre otras consideraciones de carácter inclusivo, caso del bilingüismo o el indigenismo ${ }^{39}$.

De igual modo, el nuevo sistema de selección también apuesta por incrementar la participación ciudadana, por lo que cualquier persona que satisfaga los requisitos establecidos en la Supreme Court Act puede postularse para el puesto. Para ello es necesario rellenar un cuestionario y completar una serie de formularios adicionales ${ }^{40}$.

La labor del Independent Advisory Board consiste en analizar y evaluar los perfiles de los candidatos que se presenten, así como buscar activamente potenciales candidatos que reúnan los requisitos de idoneidad. El proceso de evaluación y selección que debe llevar a cabo el Independent Advisory Board debe guiarse por una serie de criterios regulados por el ejecutivo federal. Entre ellos se encuentran tanto las cualidades personales de los candidatos como el encaje de estos en las necesidades institucionales del Tribunal ${ }^{41}$. Las cualidades individuales se dividen entre las de carácter profesional y aquellas de naturaleza estrictamente personal. En lo que respecta a las cualidades profesionales, se valoran aspectos como los conocimientos legales, la sensibilidad a los cambios sociales con trascendencia jurídica o la capacidad de análisis y resolución de conflictos jurídicos, si bien también deberán tenerse en cuenta otros factores de carácter complementario como la capacidad de síntesis y expresión, la voluntad de servicio público y la capacidad de trabajar bajo presión. Por su parte, las cualidades individuales referentes a la esfera personal del candidato versan sobre elementos como la integridad personal y profesional, el respeto, la moral, tener una mentalidad abierta o la discreción. En lo relativo a las necesidades institucionales del Tribunal, se valoran factores como el equilibrio entre expertos en derecho privado y derecho público y la experiencia legal en campos sobre los que el Tribunal esté llamado a decidir en un futuro. Junto a ello, también se menciona la voluntad de que el Tribunal represente de forma razonable la diversidad de la sociedad canadiense $e^{42}$. Por último, el panel consultivo deberá seguir las pautas establecidas por el Primer

39 Aunque el bilingüismo sea un valor añadido, este puede configurarse como una barrera de acceso al tribunal, reduciendo el número de candidatos aptos y dificultando tanto la composición territorial diversa del Tribunal como el nombramiento de candidatos pertenecientes a minorías, especialmente de las Primeras Naciones. Sin embargo, Grammond y Power; «Should Supreme Court Judges be Required to be Bilingual?» en Verrelli (ed.); The Democratic Dilemma: Reforming Canada's Supreme Court, Institute of Intergovernmental Relations, Montreal \& Kingston, 2016, pp. 49-63 refutan esta tesis en lo referente a la composición territorial, demostrando que existen jueces que reúnen las características requeridas en prácticamente todas las provincias.

40 Estos formularios pueden obtenerse en https:/www.fja-cmf.gc.ca/scc-csc/2019/form-formulaire-eng.html Los cuestionarios rellenados por los dos últimos magistrados en ser nombrados, Martin y Kasirer, pueden consultarse, respectivamente, en https://www.fja.gc.ca/scc-csc/2017-SheilahMartin/nominee-candidat-eng.html y https://www.fja.gc.ca/scc-csc/2019/nominee-candidat-eng.html

41 Terms of Reference of the Advisory Board. Disponibles en https://www.fja-cmf.gc.ca/scc-csc/2019/mandate-mandat-eng.html

42 En particular, en lo referente a miembros de las Primeras Naciones, personas con discapacidad, minorías lingüísticas, étnicas o por razón de identidad de género o sexual. De igual modo, el panel también debe guiarse por la voluntad gubernamental de alcanzar el equilibrio entre hombres y mujeres en el seno del Tribunal. A pesar de ello, en el proceso de selección celebrado en 2019 únicamente uno de los doce candidatos era mujer, mientras que solo dos pertenecían a colectivos minoritarios. 
Ministro en relación con el nombramiento, ya sean de carácter territorial, representativo o de cualquier otra naturaleza. Finalmente, durante la elaboración del informe final, que será público, los miembros del panel tienen la posibilidad de realizar consultas con el Presidente del Tribunal Supremo, una práctica ya puesta en marcha por Mulroney ${ }^{43}$, o con cualquier otra persona u organización que consideren oportuno.

Una vez terminado el proceso de evaluación, el Independent Advisory Board presentará al Primer Ministro una propuesta (shortlist), que debe oscilar entre tres y cinco nombres, con aquellas personas que considere cualificadas para el puesto. Esta propuesta no es vinculante para el ejecutivo, de manera que, formalmente, el nombramiento sigue recayendo en él y, por tanto, el nuevo sistema no conlleva reforma alguna de la Supreme Court Act o de la Constitución ${ }^{44}$. A pesar de ello, el ejecutivo de Trudeau se ha comprometido políticamente a seguir dicho procedimiento y nombrar a uno de los candidatos propuestos por el panel consultivo, pues, en caso contrario, la credibilidad del nuevo sistema, así como su oportunidad, quedaría totalmente erosionada.

Tras la recepción de la lista, el Primer Ministro procederá a elegir a uno de los candidatos, cuyo nombre será aprobado en Consejo de Ministros. Seguidamente, se dará inicio al procedimiento de audiencias ante el Parlamento previo a su nombramiento formal por parte del Gobernador General. El sistema introducido en 2016 contempla una novedad al respecto, al establecerse la comparecencia del Ministro de Justicia y del presidente del panel consultivo ante la Comisión Mixta de Justicia y Derechos Humanos de los Comunes y el Senado para que expliquen las razones que les han llevado a elegir al candidato en cuestión ${ }^{45}$. Una vez sustanciada la misma, será el turno del candidato a magistrado, debiendo este contestar a las preguntas que se le efectúen, siempre que estas no versen sobre materias que puedan ser objeto de litigio ante el Tribunal Supremo ${ }^{46}$. Este nuevo procedimiento fue estrenado con el nombramiento de Sheilah Martin en diciembre de 2017, sometiéndose la candidata a las preguntas de la Comisión mixta de Justicia durante más de dos horas, siguiéndose el mismo de igual manera en 2019 durante el proceso de nombramiento de Nicholas Kasirer.

En el plazo de un mes a contar desde la fecha de nombramiento, el panel consultivo debe elaborar un informe, el cual se hará público, en el que detalle el proceso que le ha llevado a elaborar la lista candidatos, incluyendo la personas o instituciones consultadas, los costes en los que ha incurrido y el número de candidatos que se han

43 Songer; 2008, op. cit, p. 15.

44 Russell; «Selecting Supreme Court Justices: Is Trudeau’s Sunny Way a Better Way», University of New Brunswick Law Journal, Vol. 68, n. ${ }^{\circ}$ 3, 2017, pp. 1-17.

45 El sistema introducido por Trudeau contempla que a la comparecencia pública del candidato acudan tanto miembros de los Comunes como senadores, asegurando que todas las formaciones políticas están representadas.

46 Esta restricción ha resultado en que el componente político de estas audiencias sea reducido, en claro contraste con lo que sucede en los EE.UU., evitando así que estas resulten en un menoscabo de la legitimidad de los nuevos integrantes del Tribunal Supremo y, por ende, de la propia institución. 
postulado. Además, el informe también puede ser aprovechado para proponer cambios de cara a mejorar el procedimiento en futuros procesos de selección.

Tras constituirse el Independent Advisory Board por primera vez en 2016 para cubrir la vacante ocasionada por la jubilación del juez Cromwell, se han sustanciado — hasta la disolución del Parlamento el 11 de septiembre de 2019 por la convocatoria de elecciones federales - tres nombramientos siguiendo el nuevo sistema de selección; los correspondientes a los jueces Rowe, Martin y Kasirer. Aunque todavía es pronto para poder extraer lecciones en profundidad, pues el sistema lleva apenas una legislatura en vigor, sí que se observan algunos avances en pos de cumplir los objetivos marcados por el gobierno federal a la hora de poner en marcha el mismo. La transparencia y la rendición de cuentas se han visto potenciadas tanto durante el proceso de selección, dada la publicidad del informe relativo a las tareas llevadas a cabo por el panel consultivo, como en el de nombramiento a través de la audiencia parlamentaria del Ministro de Justicia, la presidenta del Independent Advisory Board y el candidato a magistrado. El bilingüismo funcional promovido por el gobierno ha sido un criterio tenido en cuenta por el panel, resultando en la elección de tres magistrados que dominan las dos lenguas oficiales de Canadá ${ }^{47}$. En relación al resto de criterios — como la composición territorial o la diversidad - estos han influido los trabajos del panel consultivo y la lista final, contando todas ellas con candidatos que reunían dichas características ${ }^{48}$.

\section{LA PARTICIPACIÓN PROVINCIAL EN EL PROCESO DE SELECCIÓN: EL CASO PARTICULAR DE QUEBEC}

\section{La participación provincial. Un viejo anhelo}

La participación provincial en el proceso de selección de los magistrados del Tribunal Supremo es un viejo anhelo de los ejecutivos provinciales ${ }^{49}$. La articulación

47 El bilingüismo funcional hace referencia a la capacidad del juez de leer y comprender exposiciones tanto orales como escritas en inglés y en francés, así como formular y responder preguntas en ambas lenguas. A este respecto, es importante reseñar que el derecho a ser entendido por el juez en cualquiera de las dos lenguas oficiales de Canadá en los tribunales federales contenido en el artículo 16(1) de la Ley de Lenguas Oficiales (Official Languages Act) no es de aplicación al Tribunal Supremo. De los tres nombramientos realizados desde la introducción de dicho requisito, dos, Martin y Kasirer, habían nacido y estudiado — aunque en una universidad anglófona- en Montreal, mientras que Rowe, procedente de Terranova y Labrador, acreditó su dominó del francés y se comprometió a realizar cursos de inmersión para mejorarlo.

48 La composición territorial ha resultado en que los nuevos nombramientos estén condicionados por la procedencia de los magistrados salientes. Sin embargo, es importante destacar que, hasta la fecha, la función de representación territorial solo se ha predicado en relación con las provincias, quedando los territorios al margen. En relación a la diversidad, el nuevo sistema intenta poner fin al alto grado de homogeneidad en su composición que caracteriza al mismo (Songer; 2008, op. cit, pp. 26-42).

49 Choudhry; «The Supreme Court Appointments Process: Improved Federal-Provincial Relations vs. Democratic Renewal?»; Democracy and Federalism Series, Vol. 7, 2005, Institute of Intergovernmental Relations, Queen's University, pp. 1-7. 
de un mecanismo de participación provincial era un aspecto contemplado en los acuerdos constitucionales del Lago Meech y de Charlottetown. El Acuerdo del Lago Meech hubiera dotado de rango constitucional al Tribunal Supremo, modificando el artículo 101 de la Constitución de 1867 al incorporar al mismo las previsiones relativas a la composición y elegibilidad actualmente recogidas en los artículos 4, 5 y 6 de la Supreme Court $A c t^{50}$. Además, este habría incorporado al proceso la participación provincial, facultando a las provincias a proponer candidatos al ejecutivo federal cuando se produjera una vacante ${ }^{51}$. Esta participación tenía naturaleza sustantiva, pues el gobierno federal quedaba obligado a nombrar a uno de los candidatos propuestos por los gobiernos provinciales, diferenciándose entre la cuota de Quebec — en la que el candidato elegido debía haber sido propuesto por el gobierno de la provincia francófona-y el resto de magistrados cuya candidatura debía haber sido propuesta por uno de los restantes gobiernos provinciales $^{52}$. La ronda constitucional de Charlottetown continuaba la misma línea, expandiendo la capacidad de proponer candidatos a los gobiernos de los territorios, aunque la ausencia de un texto articulado resultaba en que el rol de Quebec no estuviera recogido expresamente en el mismo ${ }^{53}$.

El fracaso de ambas rondas constitucionales impidió que se materializaran estos mecanismos, aspecto que ha resultado en la inexistencia de un procedimiento reglado de participación de las provincias y territorios en el proceso de selección y nombramiento de los magistrados del Tribunal Supremo. Dicha circunstancia supone ahondar en la competencia federal en el nombramiento de jueces y magistrados, recogida en el artículo 96 de la Constitución de 1867. Aunque la mayor parte de los integrantes del Tribunal Supremo proceden de instancias judiciales provinciales, su selección para dicho cargo también es de competencia federal por lo que ni siquiera puede hablarse de una participación provincial indirecta.

Dado que la composición regional del Tribunal Supremo es un valor asentado en la política canadiense - recuérdese la convección al respecto del origen de los magistrados - podía esperarse que la reforma del proceso de selección y nombramiento articulara algún mecanismo que introdujera una mínima participación provincial. Sin embargo, la reforma llevada a cabo en 2016 no ha introducido novedades de calado con respecto a la participación provincial, quedando los ejecutivos provinciales excluidos del panel consultivo. Más allá de la previsión

50 Art. 6 del Acuerdo del Lago Meech por el que se creaba un nuevo art. 101.A y 101.B.

51 Art. 6 del Acuerdo del Lago Meech por el que se creaba un nuevo art. 101.C.

52 Aunque el art. 101.C.(1) permitía que todos los gobiernos provinciales propusieran candidatos en caso de vacante, la redacción de los apartados (3) y (4) limitaba esta facultad a las nueve provincias anglófonas en caso de vacante de un magistrado procedente del sistema de common law y exclusivamente a Quebec en el caso de que esta perteneciera al de code civil.

53 La reforma del Tribunal Supremo estaba recogida en los puntos 17 a 20 del Acuerdo de Charlottetown, quedando las partes firmantes emplazadas a abordar la participación de los pueblos aborígenes en una futura conferencia intergubernamental, cuya celebración nunca llegó a materializarse debido al rechazo del Acuerdo en referéndum. 
recogida en la norma reguladora referente a la cobertura de las vacantes pertenecientes al sistema de code civil de Quebec — circunstancia en la que es necesario adecuar la composición del panel consultivo a la tradición jurídica de derecho civil propia de dicha provincia-, la única medida tendente a introducir un mínimo grado de participación provincial ha sido la voluntad del Primer Ministro de que el Fiscal General consulte con sus homólogos provinciales sobre los candidatos incluidos en la lista final siguiendo el ejemplo de Australia ${ }^{54}$, quedando la participación provincial acotada a la fase final del proceso de selección. El gobierno de Quebec, entonces comandado por Philippe Couillard, demandó que el rol de la provincia no se limitará a dichas consultas, sino que se extendiera a la facultad de proponer candidatos a semejanza del modelo contemplado en el Acuerdo del Lago Meech ${ }^{55}$. Sin embargo, dicha pretensión fue rechazada por el gobierno federal, quien decidió proseguir con su proyecto prescindiendo de la participación provincial.

\section{El caso particular de Quebec: ¿un avance con vocación de permanencia?}

Tras el anuncio —en abril de 2019— por parte del juez Clément Gascon de su intención de jubilarse, el gobierno de Quebec, liderado por François Leagult, expresó su firme voluntad de participar en el proceso de selección en línea con el programa electoral de la CAQ. En el mismo, la formación nacionalista propugnaba que cualquier nombramiento correspondiente a la cuota de Quebec debiera producirse de entre los integrantes de una lista de candidatos elaborada por el gobierno provincial y aprobada por la Asamblea Nacional. Además, la CAQ es partidaria de que la condición bilingüe de los magistrados sea un requisito obligatorio y no meramente deseable, incorporándose el mismo a la Supreme Court Act como criterio de elegibilidad ${ }^{56}$.

54 En Australia, el Fiscal General está obligado, tal como establece el art. 6 de la High Court of Australia Act 1979, a consultar con sus homólogos estatales durante el proceso tendente a cubrir una vacante en la High Court, si bien la opinión de estos no tiene carácter vinculante. Es importante reseñar que la participación estatal en el nombramiento de los integrantes de la High Court fue un asunto controvertido durante la formación de la Commonwealth, debatiéndose sobre si la misma debía representar también a los estados o solo a la federación. Finalmente, acabó imponiéndose la visión puramente federal con el objetivo de evitar tensiones territoriales.

55 Couillard; «Une occasion à saisir», Le Devoir. 3 de agosto de 2016, p. A7, veía el nuevo sistema como una constatación de la asimetría constitucional que permite a Quebec encontrar acomodo en el seno de la federación canadiense.

56 Esta posibilidad fue tratada en octubre 2017 en la Cámara de los Comunes al respecto del proyecto de ley C-203, a través del que se proponía una modificación de los requisitos de elegibilidad contenidos en el art. 5 de la Supreme Court Act, añadiendo la obligación de comprender las dos lenguas oficiales sin necesidad de intérprete. Esta proposición fue rechazada por 224 a 65, obteniendo una mayoría favorable entre los diputados de Quebec de 37 a 31, evidenciando la diferente sensibilidad existente en la provincia respecto a este asunto. 
Confrontado con estas demandas en año electoral, el gobierno federal varió sus posicionamientos previos y concluyó un acuerdo con el ejecutivo provincial de Quebec tendente a cubrir la vacante ocasionada por la jubilación del juez Gas$\operatorname{con}^{57}$. A dicho efecto, ambas partes concluyeron un acuerdo de naturaleza administrativa — un memorando de entendimiento que carece de fuerza legal— con el que pretendían garantizar un mayor grado de participación de Quebec en el proceso de nombramiento de los tres magistrados pertenecientes al sistema de derecho civil propio de dicha provincia. Este acuerdo creaba un nuevo panel consultivo — distinto del Independent Advisory Board - específico para las vacantes correspondientes al sistema de derecho civil de Quebec: el Independent Advisory Board for Supreme Court of Canada Judicial Appointments for Quebec Seats. Ello conlleva, además, una actualización de la previsión anteriormente mencionada tendente a adaptar el panel consultivo a los nombramientos de Quebec, reglamentándose expresamente el contenido de la misma y cristalizando en la creación de un panel ad hoc para Quebec.

Este nuevo organismo está compuesto por ocho miembros —a diferencia de los siete del panel consultivo que rige para los nombramientos del sistema de common law - quienes, en consonancia con la condición francófona de Quebec, deben ser funcionalmente bilingües (art. 5 PdE). Los ocho miembros del panel consultivo son elegidos de acuerdo a la siguiente fórmula (art. $3 \mathrm{PdE}$ ): dos miembros, incluido el Presidente, a propuesta del Ministro de Justicia federal; dos miembros, de los que al menos uno no debe ser un abogado en ejercicio, a propuesta del Ministerio de Justicia de Quebec; un juez jubilado de un Tribunal Superior de Quebec o del Tribunal Supremo, originario de Quebec, a propuesta del Consejo Judicial Canadiense; un abogado del Colegio de Quebec, elegido por dicha institución; un abogado del Colegio de Quebec, elegido por la sección de Quebec del Colegio de Abogados federal; un jurista de reconocido prestigio elegido por los Decanos de las facultades de derecho de Quebec y el departamento de Derecho Civil de la Facultad de Derecho de la Universidad de Ottawa.

La composición del panel consultivo otorga el mismo peso a aquellos miembros elegidos por los ejecutivos, distribuidos a partes iguales entre el federal y el de Quebec, y los seleccionados por organizaciones independientes ${ }^{58}$. A diferencia de lo que sucede a la hora de elegir a los componentes del panel consultivo del sistema de common law, el gobierno federal tiene total libertad para llevar a cabo su elección, sin que existan prescripciones acerca de la adscripción profesional de los seleccionados. Esta sí es de aplicación al ejecutivo de Quebec — limitando el

57 La denominación oficial del mismo es «Protocole d'entente concernant le processus de nomination en vue de combler le poste qui sera laissé vacant à la Cour suprême du Canada à la suite du départ du juge Clément Gascon». En adelante, PdE.

58 La ampliación a ocho miembros conlleva que los representantes elegidos por los ejecutivos no se encuentren en minoría, si bien la división de estos entre el nivel federal y el provincial resulta en que el porcentaje de representación del gobierno federal sea menor que en el panel consultivo del sistema de common law. 
número de abogados que pueden ser nombrados- de forma análoga a como sucede con el gobierno federal en el sistema de common law. El resto de miembros son elegidos de manera similar a como sucede en el otro sistema, con la particularidad de que en lo referente a la selección del jurista de reconocido prestigio esta no se limita a las Facultades de Derecho de Quebec sino que también se incluye la sección de Derecho Civil de la Universidad de Ottawa ${ }^{59}$. Los miembros del Independent Advisory Board podrán formar parte del panel consultivo específico de Quebec en tanto satisfagan los requisitos del artículo 3, así como reúnan la condición de ser funcionalmente bilingües pues la lengua de trabajo del panel es el francés (art. 5 PdE). Salvo que el ejecutivo federal opte por lo contrario, el Presidente del Independent Advisory Board también lo es de facto del panel consultivo específico de Quebec, función desempañada en ambos organismos por la ex-Primera Ministra Kim Campbell ${ }^{60}$.

Las funciones y el mandato del Independent Advisory Board for Quebec Seats son sustancialmente idénticas a las del Independent Advisory Board en lo referente a la búsqueda y evaluación de posibles candidatos tendente a elaborar una lista de entre tres y cinco nombres. Una vez elaborada, esta será remitida al Ministro de Justicia de Quebec por parte del Ministro de Justicia federal, abriéndose seguidamente un periodo de consultas de carácter confidencial tanto a nivel federal como provincial. A nivel federal, el Ministro de Justicia celebrará consultas sobre el contenido de la lista con el Presidente del Tribunal Supremo, los ministros pertinentes del gabinete ministerial, los portavoces de justicia de la oposición, los miembros de la Comisión Permanente de Justicia y Derechos Humanos de la Cámara de los Comunes y los miembros de la Comisión Permanente de Asuntos Jurídicos y Constitucionales del Senado (art. 10 PdE). Por su parte, el Ministro de Justicia de Quebec podrá realizar idénticas consultas con otros miembros del ejecutivo, la oposición y, especialmente, con el Presidente de la Corte de Apelación de Quebec (art. 11 PdE). Una vez concluidas dichas consultas, el Ministro de Justicia de Quebec comunicará el resultado de las mismas al Primer Ministro de Quebec a quien solicitará su opinión, siendo este el encargado de transmitir la propuesta de Quebec al Primer Ministro federal. El memorando de entendimiento no hace ninguna precisión con respecto a la forma y contenido de dicha propuesta, pudiendo esta contener uno, varios o incluso ningún nombre, en caso de que el gobierno provincial entienda que ninguno de los candidatos seleccionados por el panel consultivo

59 Aunque esta universidad se encuentra en la provincia de Ontario, la misma es bilingüe, contando con una Facultad de Derecho dividida en dos secciones: common law y droit civil.

60 De igual modo, la presidenta de la ONG Centraide of Greater Montreal Lili-Anna Pereša forma parte de ambos paneles consultivos a propuesta del Ministro federal de Justicia. El resto de miembros del Independent Advisory Board for Quebec Seats fueron Louis LeBel, juez jubilado del Tribunal Supremo a propuesta del Consejo Judicial Canadiense, los profesores universitarios Eugénie Brouillet y Joseph Yvon Thériault a propuesta del Ministerio de Justicia de Quebec, los abogados Steeves Bujold y Chan-Ru Zhou a propuesta de la sección de Quebec del Colegio de Abogados federal y del Colegio de Quebec, respectivamente, Céline Lévesque por los Decanos de las Facultades de Derecho de Quebec y Ottawa. 
es idóneo para ser nombrado magistrado del Tribunal Supremo por la cuota de Quebec.

Una vez recibida la propuesta de Quebec, el Primer Ministro federal elegirá al candidato a la luz de las recomendaciones del Ministro de Justicia Federal y del Primer Ministro de Quebec (art. 13 PdE). Esta formulación ambigua responde a la voluntad de integrar la participación de Quebec sin que la misma sea en ningún momento vinculante para el Primer Ministro a la hora de seleccionar al candidato pues, en caso contrario, sería necesaria una reforma de la Supreme Court Act.

Aunque el acuerdo concluido entre los ejecutivos de Canadá y Quebec tenía la finalidad expresa de cubrir la vacante dejada por el juez Gascon, el artículo 14 del mismo extiende su aplicación a futuras vacantes correspondientes a Quebec en tanto las partes firmantes no lleguen a un acuerdo que lo sustituya. En consecuencia, el mismo tiene vocación de permanencia, manteniéndose vigente mientras no se apruebe otro acuerdo de igual o similar naturaleza. En lo referente a su modificación, cualquier proceso de reforma debe ir precedido de un preaviso de seis meses, requiriéndose el consentimiento de ambas partes para su aprobación (art. $15 \mathrm{PdE}$ ). Esta previsión refuerza la vocación de permanencia del acuerdo, impidiendo que este sea modificado o anulado de forma unilateral por parte del gobierno federal, pues, además, se declara expresamente que el mismo continuará siendo de aplicación en caso de que no haya acuerdo sobre su eventual modificación.

La canalización de la participación de Quebec en el proceso de selección y nombramiento de los magistrados del Tribunal Supremo a través de un acuerdo intergubernamental constituye un ejemplo de la flexibilidad que permite el sistema federal canadiense, en un estado cuya constitución se caracteriza por un elevado grado de rigidez, especialmente acrecentada tras el proceso de patriación constitucional culminado en $1982^{61}$. Gracias a esta flexibilidad, ha sido posible ir integrando progresivamente a Quebec en la federación con el objetivo de mantener la tensión secesionista — que tensionó enormemente las relaciones entre la provincia y el gobierno federal a finales del S.XX-en niveles reducidos.

El acuerdo firmado entre los ejecutivos federal y provincial supone un importante avance en pos del acomodo de la provincia de Quebec en el seno de la federación, materializando, de forma parcial, una de las principales demandas de la provincia francófona durante las rondas constitucionales del Lago Meech y Charlottetown ${ }^{62}$. Dicha participación es de especial importancia para el desarrollo de la identidad quebequesa pues está conectada con la idea de que la presencia de

61 En su estudio, Albert; «The Difficulty of Constitutional Amendment in Canada», Alberta Law Review, Vol. 53, n. ${ }^{\circ}$ 1, 2015, pp. 85-113, evidencia como el grado de rigidez de la Constitución de Canadá se sitúa entre los más elevados en términos comparados.

62 Dicha demanda constituía uno de los cinco puntos que el Partido Liberal de Bourassa consideraba como condiciones irrenunciables para que la Asamblea Nacional diera su aprobación al texto constitucional de 1982 . 
dichos magistrados no solo responde a la tradición civilista de Quebec, sino que también actúan como representantes de la provincia en el nivel federal ${ }^{63}$. De hecho, no debe olvidarse, tal como señala Grammond, que los tribunales y, en particular, el Tribunal Supremo, han sido las instituciones federales que se han mostrado más favorables al reconocimiento del carácter distinto de Quebec ${ }^{64}$.

Además de constituir un hito relevante en pos del acomodo de Quebec, pues estamos ante el acuerdo más importante concluido entre la provincia y el gobierno federal desde el firmado en 1978 en materia migratoria ${ }^{65}$, este tiene un gran potencial a futuro. Ello se debe a que el mismo puede servir de modelo para extender la participación de Quebec a otros procesos de selección y nombramiento, caso de los integrantes de la Cour supérieure y la Cour d'appel de Quebec o de los senadores correspondientes a Quebec. De todos ellos, el caso del Senado parece el más factible a corto plazo, pues el proceso de selección y nombramiento se desarrolla de manera similar al del Tribunal Supremo a través de un panel consultivo, cuya composición podría adaptarse para que Quebec —o cualquier otra provincia involucrada - pudiera nombrar a algunos de sus integrantes.

A pesar de la indudable trascendencia del acuerdo en pos de la integración de Quebec, el mismo no ha satisfecho plenamente las demandas tradicionales de la provincia respecto a los nombramientos en el Tribunal Supremo ${ }^{66}$. Esta cuestión fue recordada por el propio Legault, quien calificó el acuerdo como un primer paso hacia la articulación de un sistema que permita a Quebec proponer directamente los nombres de aquellos candidatos de entre los cuales el gobierno federal deberá realizar el nombramiento ${ }^{67}$. Sin embargo, cualquier reforma en este sentido, debería pasar por una modificación de la Supreme Court Act, circunstancia que no parece posible en un futuro próximo debido a las dificultades crónicas de las que adolece Canadá para reformar su Constitución ${ }^{68}$.

63 SCHERTZER; «Quebec Justices as Quebec Representatives: National Minority Representation and the Supreme Court of Canada's Federalism Jurisprudence», Publius, Vol. 46, n. ${ }^{\circ} 4$, 2016, pp. 539-567.

64 Grammond; «Louis LeBel et la société distincte», Les Cabiers de droit, Vol. 57, n. ${ }^{\circ}$ 2, 2016, pp. 251-267. Acerca de dicho reconocimiento, en relación a la importancia de la lengua francesa como elemento nuclear de la sociedad quebequesa vid. Nguyen v. Quebec (Education, Recreation and Sports), 2009 SCC 47, [2009] 3 S.C.R. 208, par. 40.

65 El acuerdo Cullen-Couture permitió al gobierno de Quebec asumir, a través de un sistema de vetos positivos y negativos, competencias en relación con la selección de inmigrantes. KosTov; «Canada-Quebec Immigration Agreements (1971-1991) and Their Impact on Federalism», American Review of Canadian Studies, Vol. 38, n. ${ }^{\circ} 1,2008$, pp. 91-103.

66 La Asamblea Nacional aprobó en 2013 una moción en la que reiteraba su postura tradicional al respecto de que los tres jueces de Quebec sean nombrados entre los miembros de una lista de candidatos elaborada por el gobierno provincial (Journal des débats de l'Assemblée nationale — 40e législature, 1re session, mardi 29 octobre 2013, Vol. 43, n. ${ }^{\circ} 84$, pp. 5208-5209).

67 Chouinard - Marquis; Cour suprême : une entente « importante », mais " pas idéale », dit Legault, La Presse, 15 de mayo de 2019. Disponible en https:/www.lapresse.ca/actualites/politique/201905/15/01-5226168-cour-supreme-une-entente-importante-mais-pas-ideale-dit-legault.php

68 En relación con la dificultad para reformar la Constitución de Canadá, así como sus diferentes procedimientos según las materias afectadas, vid. Pelletier; La modification constitutionnelle au Canada, Carswell, 


\section{REFLEXIONES FINALES}

La reforma del proceso de selección y nombramiento de los magistrados del Tribunal Supremo de Canadá puesta en marcha por el gobierno de J. Trudeau como parte de su paquete de medidas para mejorar la calidad democrática responde a la voluntad gubernamental de potenciar la legitimidad de las instituciones, aumentando su transparencia y fomentando la rendición de cuentas. Este paquete de reformas viene a continuar un proceso de tímida reducción del dominio por parte del ejecutivo del proceso de selección y nombramiento, iniciada en 2004, con la apertura del proceso a la participación del Parlamento. Sin embargo, la ausencia de un procedimiento reglado ha impedido que la participación del Parlamento se consolide, viéndose enturbiada por la lucha partidista.

Al igual que ocurrió en el caso del Senado, el ejecutivo ha optado por la vía infraconstitucional para poner en marcha sus reformas, evitando variar formalmente el poder de nombramiento que sigue recayendo en el Primer Ministro, pues cualquier alteración de los elementos esenciales de la institución requeriría seguir los mecanismos de reforma constitucional tal como aclaró el propio Tribunal Supremo al reconocer la naturaleza constitucional de la SCA.

La creación de un panel independiente de expertos con la misión de identificar y evaluar a potenciales candidatos para el Tribunal Supremo supone un importante avance a la hora de mejorar la legitimidad de la institución, pues se reduce el control político sobre los nombramientos, basándose estos en criterios jurídicos y profesionales. La composición del panel, con mayoría de expertos jurídicos y en el que los miembros seleccionados por el ejecutivo son minoría, es otro factor que contribuye a potenciar la independencia, transparencia y legitimidad del proceso de selección. Aunque la propuesta que elabore el panel consultivo no es jurídicamente vinculante - para evitar tener que llevar a cabo una reforma constitucional—, sí que lo es en el plano político pues, en caso contrario, el nuevo sistema no sería viable. Además, pese a su implantación reciente, el nuevo modelo parece ir consolidándose gradualmente dado que la oposición ha formado parte activa del mismo a través de las audiencias parlamentarias, no habiendo expresado objeciones que hagan peligrar su continuidad en el futuro de forma contraria a como ha sucedido con la reforma del sistema de designación de los senadores.

La participación provincial en el proceso de selección continúa siendo una de las cuestiones pendientes desde el fracaso de las rondas constituciones del Lago Meech y Charlottetown, sin que la reforma de 2016 haya conllevado novedades al respecto. En lo referente al caso particular de Quebec, el acuerdo concluido entre el ejecutivo provincial y el federal supone un primer paso para articular la participación de esta provincia en el proceso de selección a través de la composición del panel independiente. A pesar de la naturaleza administrativa y, por

Toronto, 1996. 
tanto, no vinculante del acuerdo, su vocación de permanencia y la imposibilidad de modificación unilateral de este, se configuran como garantías de la continuidad del sistema, el cual viene a dar respuesta a una de las demandas tradicionales de la provincia francófona, pues el desarrollo y salvaguarda del sistema de derecho civil propio de Quebec constituye uno de los elementos centrales de la identidad quebequesa como el propio Tribunal Supremo reconoció en el caso Nadon. Aunque este acuerdo no haya satisfecho completamente las demandas de Quebec, pues no le permite proponer candidatos, sí que constituye el mayor avance tendente a mejorar el acomodo de la provincia dentro de la federación canadiense en los últimos años, pudiendo servir de modelo para otras instituciones, así como base para una reforma constitucional que reintegre, de una vez por todas, a Quebec en el consenso constitucional post 1982.

\section{$* * *$}

TITLE: The reform of the process of selection and appointment of the justices of the Supreme Court of Canada: the case of Quebec

ABSTRACT: This paper's aim is to analyze the recent reform of the process of selection and appointment of judges of the Supreme Court of Canada. After analyzing the constitutional nature of the institution, it covers aspects such as its composition and eligibility, including the role of the federal Parliament. Following this, the system of selection and appointment is studied with a special focus on how it can channel the participation of Quebec, achieving one of the traditional demands of that province.

Resumen: Este artículo analiza la reciente reforma del proceso de selección y nombramiento de los magistrados del Tribunal Supremo de Canadá. Partiendo de un análisis sobre la naturaleza constitucional de la institución, se abordan cuestiones acerca de su composición, con especial énfasis en las funciones desempeñadas por el Parlamento federal. Con esta base, se estudia el nuevo sistema de selección y nombramiento, prestando especial atención a cómo este ha servido para articular la participación de Quebec en el mismo, materializando una de las demandas tradicionales de dicha provincia.

Key words: Supreme Court, Canada, Quebec, Federalism.

Palabras Clave: Tribunal Supremo, Canadá, Quebec, Federalismo.

FECHA DE RECEPCIÓN: 18.12.2019 FECHA DE ACEPTACIÓN: 09.09.2020 